\title{
Design, Structure and Significance of Virtual Machines in Network Management System: An Emulation Analysis of VMware Based Hospital System in Pakistan
}

\author{
Umair Shahzad ${ }^{1,2}$, Muhammad Luqman ${ }^{1}$, Rao Muhammad Adnan ${ }^{1}$ \\ ${ }^{1}$ Department of Computer Sciences, Virtual University of Pakistan, Lahore, Pakistan \\ ${ }^{2}$ Department of Mass Communication, Forman Christian College (A Chartered University), Lahore, Pakistan
}

Email address:

purpleresearchfeedback@gmail.com(U. Shahzad)

\section{To cite this article:}

Umair Shahzad, Muhammad Luqman, Rao Muhammad Adnan. Design, Structure and Significance of Virtual Machines in Network Management System: An Emulation Analysis of VMware Based Hospital System in Pakistan. Automation, Control and Intelligent Systems. Vol. 8, No. 2, 2020, pp. 13-23. doi: 10.11648/j.acis.20200802.11

Received: July 22, 2020; Accepted: August 13, 2020; Published: August 27, 2020

\begin{abstract}
The trend of the use of virtual machines is vitally growing in the world. Virtual machines are being used in different organizations. These virtual machines come as a result from an output of a host hypervisor. If an organization or an institution is being run by virtualization then it is possible that unnecessary expenses, additional burden, wastage of resources and excesses of space can be reduced for networking. With the organizations when the structure of a medical institution or hospital is viewed it comes out that even big and small hospitals are shifting their management systems to certain kinds of networks. As it is visible in the rapidly advancing world that hospitals are also using networking technology so why not they shift to virtualization? So, to observe either virtualization can effectively run in a hospital scenario this research is conducted. To further analyze if this can work in the setting of Pakistan or not? This research is conducted according to an assumed situation of Hospital that has four different branches in a country. To find the outcomes simulation method is used. A network is built on VMware Workstation. This network is designed to run a hospital networking system. That comprises of a virtual private network server, firewall router, file transfer protocol server, web server, and voice over internet protocol server, virtual local area network, active directory, virtual switches, dynamic host control protocol et cetera. After proper testing of the VMware then fully loaded system is launched and every functional requirement is utilized to clearly investigate the progress of a virtual network and this research has resulted in a successful network management system for Health Care Hospital in Pakistan. In viewing this the researcher has invented two concepts in this research that are "Hospital Virtualization" and "Pocket Network Theory."
\end{abstract}

Keywords: VMware, Virtual Machine, Emulation, Hospital Virtualization, Simulation Method, Network Security, Computer Science, Pocket Network Theory

\section{Introduction}

Network is a significant tool in an organization. It connects different points within the internal infrastructure and external branches of a company. Network has always been a challenging system for a company because as the technology advances the previous systems entirely transform. Nowadays layer of virtual networking is vastly spreading in the technological market. In western countries many different organizations are shifting their large messed up network rooms to small highly advanced upgraded servers and turning their networking traffic from LANs to VLANS [12]. This kind of networking environment is also being introduced in Pakistan. Many small-scale organizations are now implementing this paradigm and gaining benefit from virtualization. Network is even used in hospitals it has a vital work to do. Now as the technology is available to reduce space for network related systems. There is no best place than a hospital to start from that can result in the compression of network room space and expansion of more area that can be utilized by the doctors for patients. Here in this research we have assumed that Health Care is a hospital that provides medical facilities to patients. It has many branches throughout the country. Main branch is in Peshawar and the sub branches 
are in Islamabad, Karachi and Lahore. To automate the hospital system, we have used Client Server architecture.

There are various employees as Medical Superintended, doctors, administrators and clerks having different duties according to their scales. All branches are combined via functional network that shares information and make audio video calls time to time when required. The hospital has a web server having different pages that holds hospital, employees and patient records that is accessible through web browser from any branch of the hospital. Web server has a main page with hospital logos and some relevant design pictures. Staff web page has necessary record of the employee name, address information, post, and scale salary. Patient web page has details of patient info as, treatment history. Important data and information files are shared through file transfer protocol. Servers are set between the branches. The file transfer protocol server is installed in main branch. As all the branches reside in remote geographical locations and internet is used to connect all branches so a secure connection is built for traffic between all branches by configuring virtual private network and Firewall between the main branch and sub branches. The main branch has domain controller. User accounts login and access rights are managed by domain controller. Additional domain controller resides in Islamabad branch. Member Servers are installed in Karachi and Lahore. Network Engineer will be available for providing computer related facilities. Computer trouble shooting means diagnosing, finding, maintaining and repairing computing related devices [4].

\subsection{Objective of the Research}

To test if VMware Workstation can be an authentic tool that can facilitate proper networking mechanism via virtual machines in a hospital.

\subsection{Significance of the Study}

This research is being conducted to view the benefits of VMware as a tool that can facilitate a hospital system with less space, man work, resources and cost. So that the usability of the system can be fully analyzed. In software engineering, usability is the degree to which software can be used by a specified consumer [7].

\subsection{Hypothesis}

$\mathrm{H}_{1}$ : VMware Workstation can be a significant tool for virtual networking in the Hospitals of Pakistan.

\subsection{Simulators Review}

VMware Workstation is a product that enables systems, network or computer administrators to build and run many different virtual machines by the use of a single host physical device. These virtual devices work parallel at the same time without the interruption from other VM machines and even the physical host hypervisor also runs independently without impacting the VM machines. Each VM runs its own OS. VMware vSAN tool provide storage that is software oriented and is installed on the ESXi hypervisor and connected with vSphere, it pools with different disk capacities from many ESXi hosts and provisions it through smart policies, for example erasure coding, protection limits and thin provisioning [13].

VMware Workstation has the ability of Creative Labs 16 Sound compatible audio device and supports in Windows, NET Server and Linux guest operating systems. VMware Fusion can enable one or more cameras on virtual device. The VM uses the camera defined as the default. If you have multiple cameras on the host system you can choose another camera for the VM usage.

The logic behind not having or using most of simulators is that it cannot have that many options to tackle such big and difficult systems. But VMware has the ability to do so whereas other common simulators or emulators do not have such ability. To compare the characteristics of simulators suitable for this research some of the simulator's details are mentioned as free network simulators are based on their own parameters that are CPU usage, memory usage, computational time and scalability. Some of the commonly used network simulators and their comparison are below but still they lack many functionalities required by different networks.

The simulators that are Mininet, Cisco Packet Tracer, and emulator GNS3 and EsitNet lack the ability of running a fully loaded network of a hospital. Firstly, we should know how different simulators and emulators are; because emulator works as an actual copy of network operating system. Whereas, simulator just show specimen work instead of actual network OS. The actual problem is to analyze the performance results of network transmission for these tools. The problems comprise of scaling to large networks, testing the correctness and evaluating the performance with the ability to easily migrate to an actual system with fewer changes for deployment. GNS3 simulates entire networks, not just network OS. Many networkers use GNS3 for emulation of IOS. For example, Cisco et cetera. But GNS3 is different from other simulators because of functionality to emulate routing and switching and incorporate. It also incorporates VMs and joins together via tunneling protocol. But GNS3 is not feasible at running switches so it becomes difficult to run a fully functional network on it [9].

Packet Tracer simulator only provides specimen functionality of equipment features it is not an actual emulator. Nowadays Packet Tracer is only used for the analysis of network scenarios in increasing and becoming a demand-oriented simulator for the testing of networks prior to release [10].

In today's scenario it is highly significant to execute experiments before the whole network to avoid wastage of funds, to have benefits, to reduce the complexity of management and to keep hold of the additional resources that are needed for the network release related concerns [2].

MiniNet builds Openflow protocol network that is virtual including a controller. This simulator allows forming customized topologies via links, hosts and switches. [9].

EstiNet simulator can be used for many different controllers and it has the ability to work in both emulation mode and simulation mode. The disadvantages of EstiNet comprises of lack of research material and studies on the internet. It is not popular enough in general and has very less or no specifications towards [3]. 
After viewing different specifications, I can come to a conclusion on the evaluation of the emulators and simulators that EstiNet, OpenFlow and other network simulator and similar emulators are not the actual virtual systems provider of information systems market on the basis of which research cannot actually be conducted [9]. The upcoming table presents specifications-based comparison of different emulators and simulation software including VMware, GNS3, Cisco Packet Tracer et cetera. Further table show the abilities of some of the simulators and emulators and their limitations:

Table 1. Network Simulators Features Comparison [9].

\begin{tabular}{|c|c|c|c|c|}
\hline General & Mininet & Packet Tracer & GNS3 & EstiNet \\
\hline Free software & Yes & Yes & Yes & No \\
\hline Open source & Yes & No & Yes & Yes \\
\hline Publicly downloadable & Yes & No & Yes & No \\
\hline Windows support & No & Yes & Yes & No \\
\hline Linux support & Yes & Yes & Yes & Yes \\
\hline Fully functional IOS & No & No & Yes & No \\
\hline Emulation mode & Yes & No & Yes & Yes \\
\hline Compatible with real world controllers & Yes & No & No & Yes \\
\hline Result repeatable & No & No & No & No \\
\hline Scalability & Middle by multiple processes & No & No & High by single process \\
\hline Performance result correctness & Depend of resources & No & Yes & Yes \\
\hline Wifi & Yes & Yes & No & Yes \\
\hline Gui support & Yes & Yes & No & No \\
\hline
\end{tabular}

After viewing the information available with the experts and internet it can be evaluated that abilities of different simulators in the context of diverse parameters are very limited and cannot run networking systems as VMware can do. Packet Tracer cannot provide actual networking system as it is just a specimen for training, GNS3 is based on Dynamips and QSEMU works on actual IOS images but the major drawback of GNS3 is that it utilizes too much resources of CPU available on the computer and virtual machines for clients $\&$ servers simulation. Mininet specifications comprises of unique open-source network simulator that is developed to support research and education in Software Defined Networking, It is viewed that Mininet command-line interface is easy to use but cannot run actual networks as VMware can, the logic behind this is that Mininet uses network namespaces as its virtualization technology, it cannot actually assist for a large number of VLANS without reducing down the simulation, and NetSim does not have a proper IOS and it only runs some of Cisco routers but not every router.

\section{Methodology}

The adopted methodology in this research is simulation method because as it is different than other fields of life so here out of frequently used methods of computer sciences this method is being used.

\subsection{Simulation Method}

It is practiced specifically in computer science as it provides ability to examine networks that are outside of the experimental domain or in other context the networks that are not yet invented. Frequently difficult concept, those with rare ability of implementation in actual situation. Few fields that opt for computer-based simulation methodologies are sciences such as astronomy, economics or physics; other areas are specialized such as the studies of virtual actuality et cetera. also exploit these methodologies. Many networkers use this method, as it provides options of doing new research on developing network protocol. For testing the network, organization or an individual will build a wide network after the investment of costly network tools, and it is not possible enough to easily achieve it. So as per the fact, simulation method can be used [15]. Network simulation methodology is a beneficial and frequently used methodology for evaluation of diverse networks without their implementation in the world. Here objective of the research comprises of a plan for generating a hospital network that can work to enable audio/video calls, share information, transfer files (patient reports, employee details et cetera.) and set security parameters for the different categorization of the employees in the hospital by the domain controller. The software VMware Workstation is used and via simulation method four branches of a hospital is built via networking system and on that network the official work of hospital is further carried out to see the performance of the virtual network, extended star topology is used.

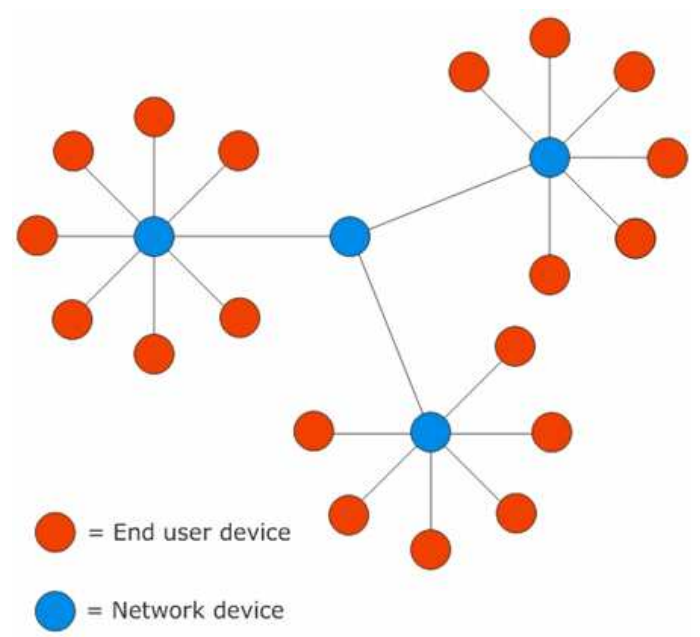

Figure 1. Extended Star Topology. 


\subsection{Network Topology}

It is defined as organization of a network. It includes nodes and joining lines. The structure on which the topology that is used in this research is extended star Topology. The logic behind it is extended star topology it joins more than one different star. The use of extended topology is to generate large local area networks and even on the other hand every extended star can be located in different locations in a WAN. In this research as there is one main site and other three remaining are the sub-branches so Figure 1 is a topology specimen on the concept of which a network design of this research is made.

\subsection{Client-Server Architecture}

This architecture is used because it is the best suitable one for the requirements of this research. In this model client demands a service from a server over the internet. Here, Client serves as one set of program/code which executes a set of actions over the network. Whereas, whole server represents a separate program, that sends outcomes to client system. The client computer sends an interface to user for service or a resource and server processes the request and displays the results to user. The host hypervisor and VMware run as a platform of the system. Below is the Client-Server Architecture design of this research:

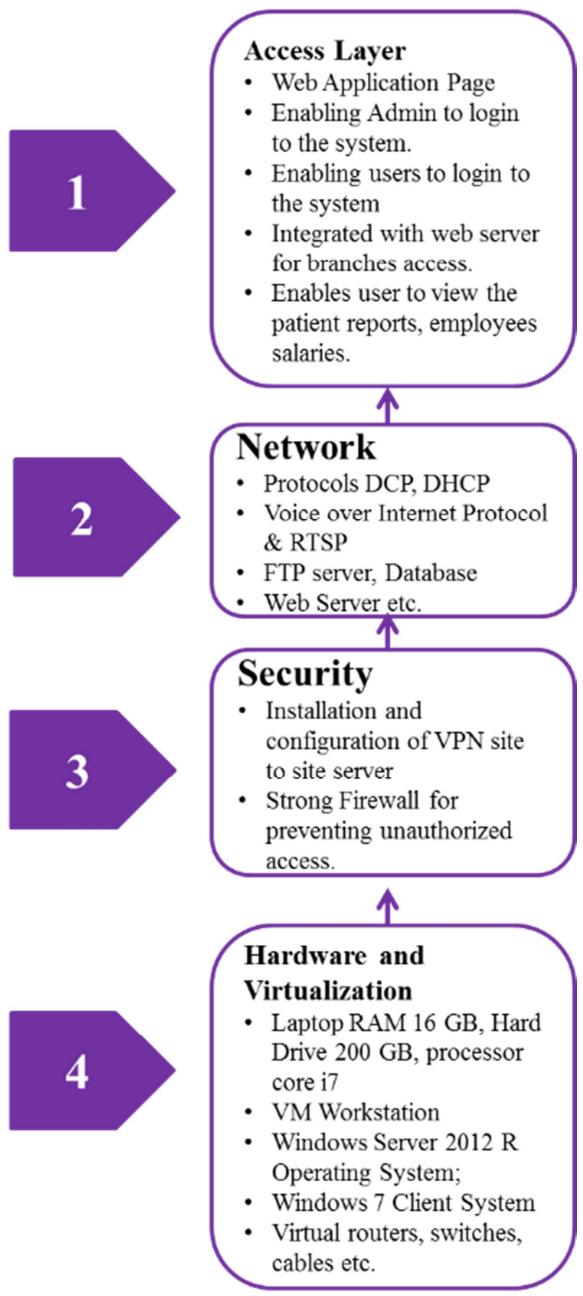

Figure 2. Client-Server Architecture Design.

\subsection{VMware and Networking Process}

As a network management system comprises of elements of the network [1]. So here in this research "VMware Workstation Pro 12" is used to install virtual machines and show them as servers of different branches of a hospital and then work according to the requirements of the research. As the research comprise of four entirely different sub-domains including network domain, web server, audio/video calls communication, data transfer and user login parameters definition so only any single simulators cannot be possibly able to carry out this network management research for example a routing simulator might not able to do video call so for that to do a combined networking research different virtual machines are required that can be set in a single physical device and to do that VMware applications and different products are useful source because VMs comprises of virtual networking with security and management tools, software-oriented data center with storage capacity software. In VMs process, a host hypervisor is installed on an actual server that allows multiple virtual machines to run on the individual server. Every $\mathrm{v}$ machine has the ability to run its OS. These VM share memory, for example RAM. Different VM applications can be installed together in a single physical device and can be integrated together for the research.

\section{Proposed System}

To run a proper network a proposed system is required with the specifications that are according to the requirements of the system. If the network is not built on the proposed requirements then it is possible that the system can collapse which may cause failure of the research. For this research certain requirements are set on the basis of which the system will be set.

\subsection{Functional / Non-Functional Requirements}

A complete functional and secure network is built for the given scenario. Virtual local area networks are made for different staff members, User accounts are created for login for different staff members of the hospital. Access policies are made for staff members of the hospital. Technical details include Installation and configuration of VPN server and Firewall, Installation and configuration of the DNS, Installation and configuration of the DHCP, Installation and configuration of the web server, Installation and configuration of the FTP server, Enabling Admin to login to the system, enabling users to login to the system. The logic behind using VMware is that many appliances are designed to run in VMware because desktop VMs are more responsive in VMware than other simulators and emulators [8].

The non-functional requirements comprise of actually two devices that are assigned for this research because if one is not in working so backup device can be used to cover the working of the research. The primary device that is formally 
used for this research is Windows 7, Professional 64-bit, English, System Builder: Dell Inc., Structure Model: Precision WorkStation T5400, Basic Input Output System: Phoenix BIOS Read Only Memory PLUS 1.10 Version Processor A04: Intel Xeon (R) CPU X5450@3.00GHz (8 CPUs), 3.0GHz, Memory: 32768MB RAM. Hard Disk 1TB.

The backup device that is used for this research is Windows 7 OS laptop having 16 GB Random Access Memory, processor core i7, $200 \mathrm{~GB}$ hard drive and windows 7. The details include English Windows 7 Professional 64-bit, System Builder: Hewlett-Packard System Model: HP Elite Book 8470w: Default System BIOS Processor: Intel Core 17-3630QM CPU; $2=2.40 \mathrm{GHz}$ (8 CPUs), 2.4GHz Memory: 16384MB RAM. Hard Disk 200 GB.

There are networking accessories as Virtual, switches, cables that are included and Software tools that are required by the system includes Network emulator that can be used that fully supports the research requirements and that is VMware Workstation. For servers IOS Window Server R2 2012 is installed. For client Windows 10 is installed. For File transfer protocol http over ftp application is installed. For reading the files Adobe Reader is installed. For web server XAMPP server is installed. For audio, video calls and communication output messenger are installed.

\subsection{Textual, Audio and Visual Communication}

The calling facility is easily possible by Horizon Virtualization Pack for Skype for Business via Virtualizing Skype for Business with Horizon with that appropriate user experience with Microsoft and VMware's optimized Skype for Business solution can be carried out [6]. But as Skype for Business is paid software and as this is a student research and within limited resources the student has to deliver so for implementation output messenger is used it has the ability to create an administrator and then the users. Output messenger is used to have voice over internet protocol work for the network management system. It has the ability to send instant chat messages, screen sharing, audio calling, video calling et cetera. It is also secure as Skype because it will run on a secure network outsider will not be able to access the output messenger. Output Messenger is the Private Instant Messaging application which has all communication features like Instant Messaging, Group Chat, Announcement, Chat Room, Voice and Video Call et cetera. in one pack with Screen Clipping, Notes, Share Notes, Internal Mail, Reminders [14].

\subsection{Throughput and Delay}

Actually, as VM comes under virtual environment so the readings will remain average in the calculation but can be calculated by using any general networking tool for example in this networking scenario software "IPERF" is used that runs in command prompt. This application immediately shows the throughput, delay, bandwidth, packet count et cetera of the networking traffic.

\subsection{Virtual Private Network}

VPN technology generates a secure and encrypted connection using less defending network, for example internet. This technology was developed to allow remote users and branch offices to securely access corporate oriented data and sources. In this research pfsense software is used to generate site to site VPN. Here in the provided scenario point to point VPN connections between the four offices are used and IPsec is used. To check as evidence that either the network traffic is being encrypted via Firewall or not the software "WIRESHARK" is used.

\subsection{Router and Firewall}

Firewalls it is for the security of network so that the network is not compromised, different hackers trying to gain access to network from the outer world. Firewalls are actually hardware devices but nowadays it is available in soft form, so that can be installed on a device. Here pfSense is used as a Firewall. It is a no cost firewall that comprises of router for Security protection in a network [11].

To establish a connection between four offices virtual firewalls are used that have the ability to work as routers too. These firewalls can be up to sixth layer devices too. But here in the provided scenario pfsense firewalls are used. pfSense is a free firewall and router based on FreeBSD. pfSense can be installed on a virtual machine or actual computer to make a dedicated firewall and router for a network as per the required network both routers and firewalls are required so it covers both of the requirements.

\subsection{Security and Access Control}

Security and Access Control in user interface is necessary for policy-based network. A web application is used for the network. In the field of computer sciences website is a client-server computer program that the client runs in a browser. The website is used so that rights, access for the security point of view can be specified for the users. Below is the specimen of the login screen that provides access to the system when a domain controller or a user login to the screen. It only opens the application once the login id and password are correctly provided to the application. As per the provided instructions the domain controller at main branch and additional domain controller in sub branch are installed with internet protocol configurations network screens are shared further: Point to point tunneling protocol is used to connect the branches via a secure network. IPsec tunnels are generated in the firewalls. For servers static routing is used whereas for user dynamic host control protocol is used for network configurations.

\subsection{Web Server}

A web server named "XAMPP" is used for hospital record. XAMPP is defined as Cross-Platform denoted by "X", Apache A, MariaDB M, PHP P and Perl P. In simpler words, lightweight Apache distribution makes easy for coders to 
generate web server for deployment and examining purposes [16]. It is built on PHP with MySQL database where the proper working of hospital takes place. It is significant to involve accurate designing of the system that will be implemented for a specific implementation. So, it is recommended to give a professional quality overview of the design [5]. In the results section the graphical user interface is provided.

\section{Testing}

Testing is a significant part of the research where you concentrate on investigation and discovery. During the testing phase, networkers find out whether their network and systems work according to customer requirements. And while it's not possible to solve all the failures you might find during the testing phase it is possible to use the results from this phase to reduce the number of errors within the network program. Before testing can begin, the networkers develop a test plan. The test plan includes the types of testing that is used, resources for testing, how the network is tested, who should be the testers during each phase, what are instructions each tester uses to test the network.

This phase is vital for network development because a provided scenario requires checking prior releasing the network It is important to have a testing engineer that can network by putting effort and knowledge for surety that the network has no defects. Network checking points errors that happen during the development phase. Tester makes sure that the network's performances are adequate and that customers have satisfactory responses. Network testing with strict test execution assures lower maintenance cost. A critical defect left undetected can cause major problems in business and then owner might have to pay big price of the loss. Every serious company will invest in a test phase and find experts who will be capable of detecting problems and flaws in a networking system.

The benefits of Network Testing comprise of reduction in cost of whole system that may be caused if the network have errors by testing because, their goal is to make the accurate network possible. Benefits of network testing are huge and they have a significant role in entire business. The quality of a product is high, and that is important for the customer. Even more important is that with selling high-quality products, Network needs to be simple, understandable, and easy to use. Only testers can assure that. Their experience will make sure that the network is designed in a way that is logical. If an appropriate user experience is required, network needs to be free from errors, which can be a source of problems for users. Choosing a feasible network testing service with a professional group will guarantee the quality of a product and authentic user experience. The major benefit is that network testing leads to business optimization. Business optimization means satisfactory clients, customer retention and fewer costs of fixing a product, fewer costs of a customer service, quality and validity of a network.

In this research initially minute requirements are set to further build the whole network. The requirements comprise of installation of network emulation or simulation tools. As the tools are optional in this project VM Workstation is used as an emulation tool. Domain controller is set at main branch and additional domain controller is set in sub branch. Two routers and firewalls are established for connection between two offices. Point to point virtual private network connections between the two offices is built. By active directory the permission for allowing users is set to connect or login remotely. Shared folder is set for the transfer of data between offices and enabled remote desktop connection between domain controller and additional domain controller. Here according to the provided scenario network is built and further testing results are clearly shown as per the requirements in screenshots so that evidence of the instructions can be highlighted in the actual results of the research.

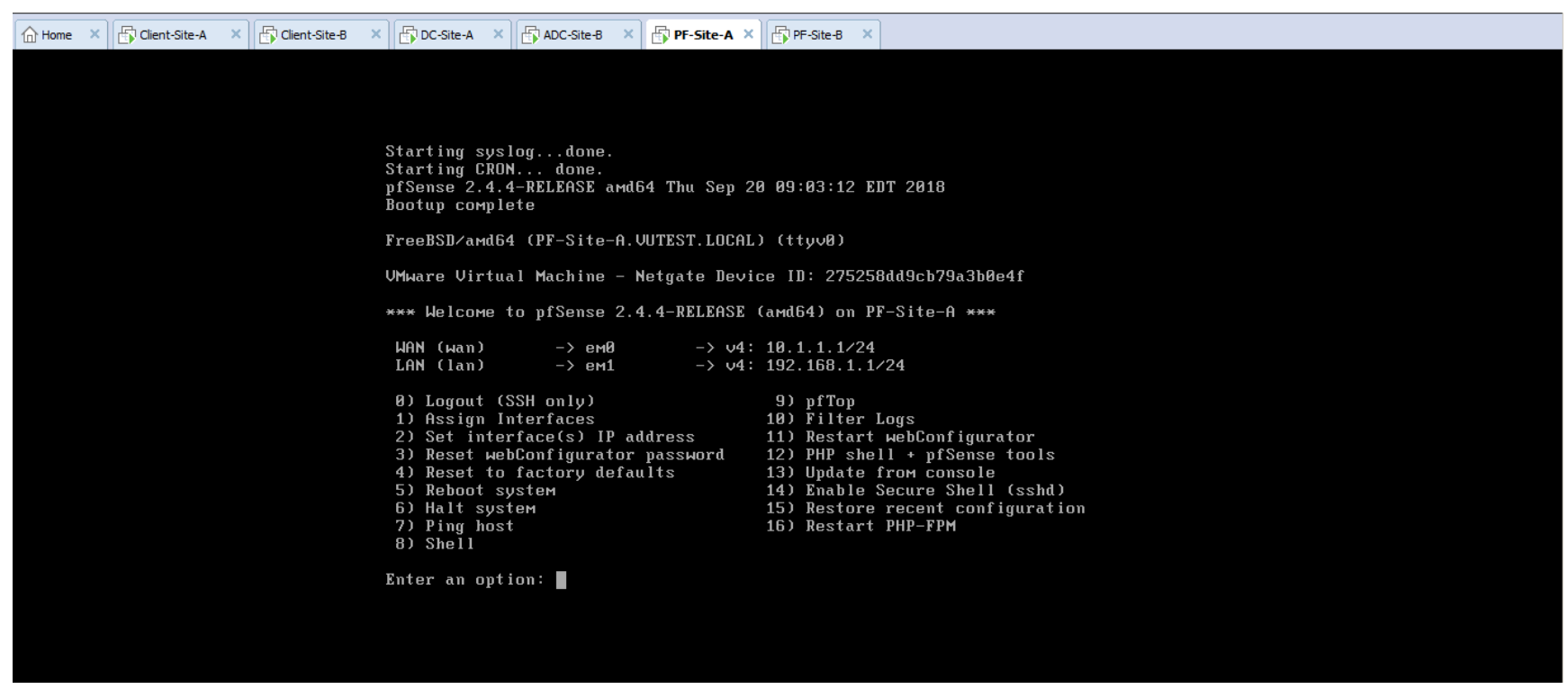

Figure 3. PFsense-Router/Firewall Peshawar. 


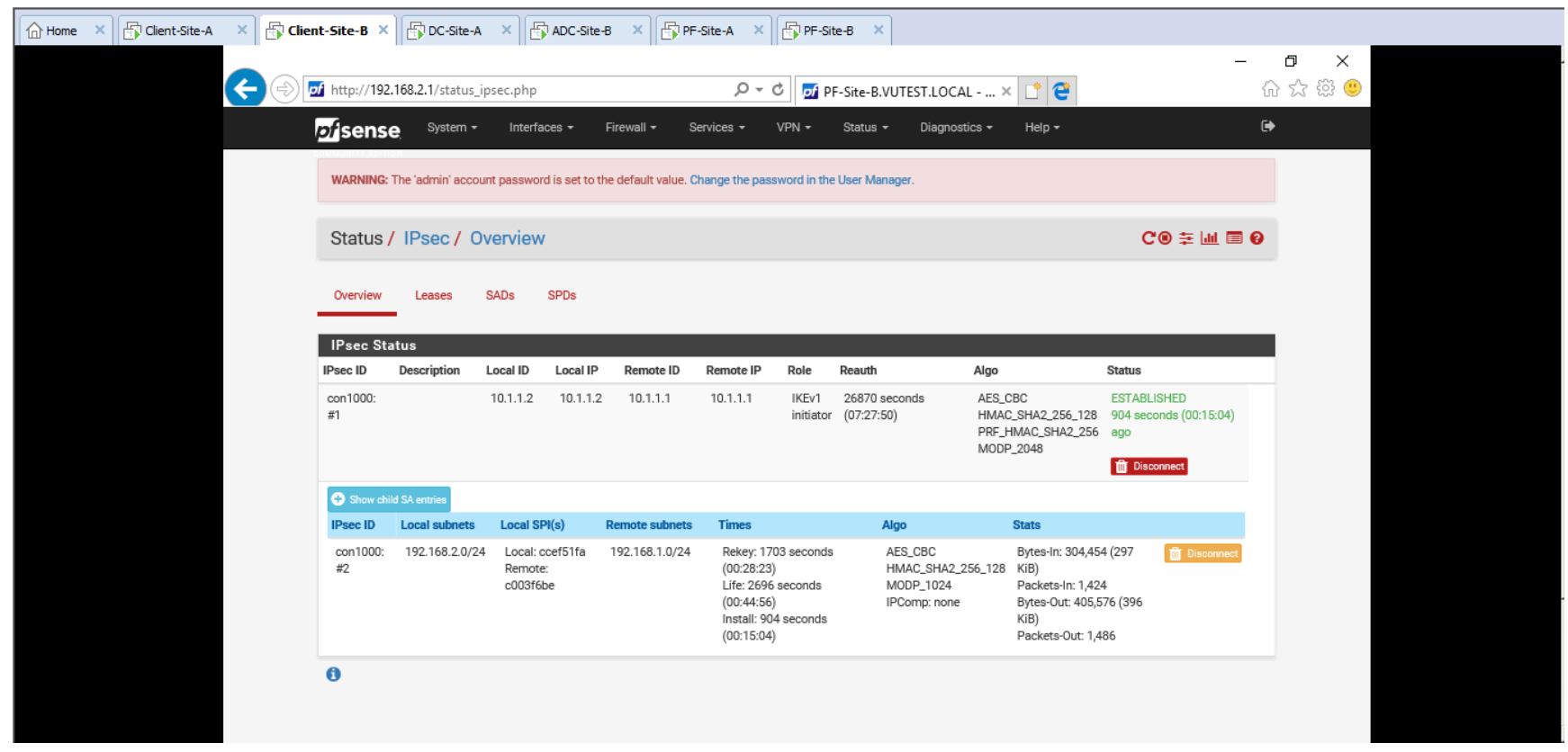

Figure 4. Additional Domain Controller-Site-B IPsec point to point VPN.

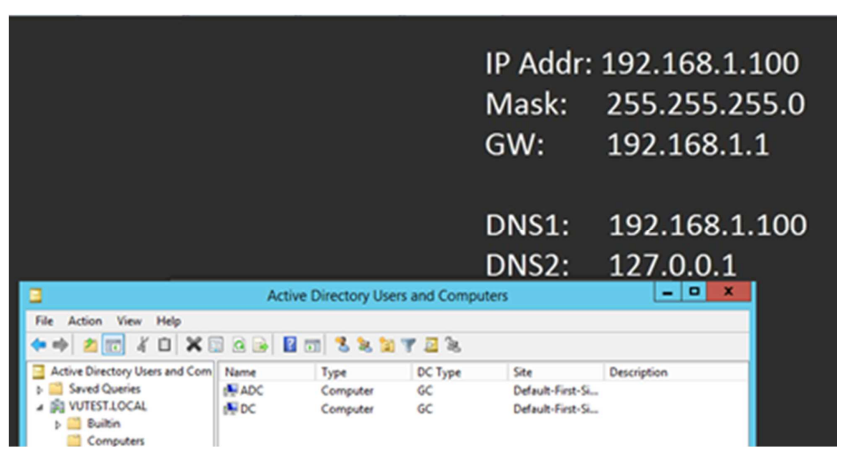

Figure 5. Domain Controller and Active Directory View.

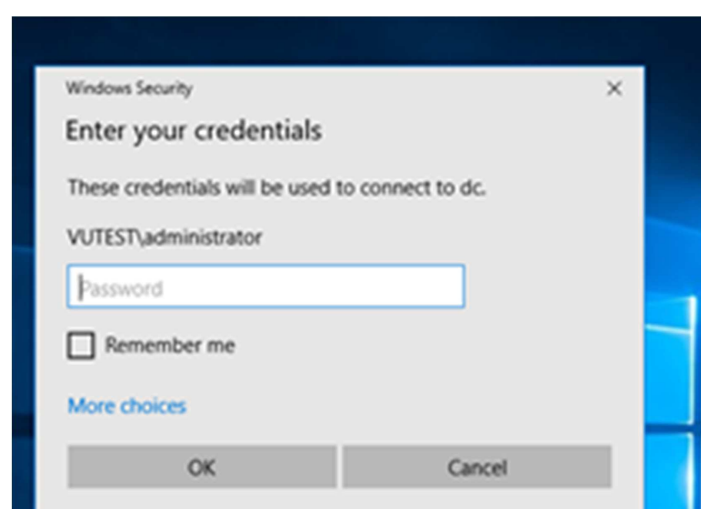

Figure 6. Remote login credentials screen accessing D.C.

\section{Conclusion and Implications}

After when the testing is successfully achieved now further whole network is designed and developed where fully functional requirements are transformed into actual network. A domain controller is set in the Peshawar branch. Firewalls and routers are placed in each branch. With different applications it is insured that hospital system requirements are achieved and users are able to work accordingly even an additional domain controller is set too as a backup and each site is fully functional on the network. Further functionalities are analyzed to prove either the network system is feasible for a hospital or not. As the research requirements are visible after properly using the network in the system. Hence, $\mathrm{H}_{1}$ is accepted in the setting of Pakistan that VMware Workstation is a significant tool for virtual networking in the hospitals of Pakistan. The conclusion is proved that virtualization can take place of physical networks and reduce costs, space, time, man work et cetera in a small setting where it is difficult to invest and hard to maintain the functionality of a hospital. With this system the record of a hospital can be properly secured and maintained that can reduce errors, loss of data and much such problems that can cause patients, doctors, clerks and administrators et cetera to suffer while doing what is required for the working of hospital and in the context of implementation if any hospital in the beginning stage requires a network for specimen-oriented scenario, the hospital representatives can use this network management system for testing and the system will definitely be able to fill the requirements of a hospital and will become a path to the actual physical network of a hospital. As the research has successfully shown the operational network and the advancement in the networking field is reducing space and enhancing functionalities, so the researcher predicts a future theory in his research named "Pocket Network Theory." It is defined as a network run by a small pocket size device that is paper thin and can allow a networker to monitor the network wirelessly where ever the network engineer is in the world by the combination of VLANs and even the destination devices of the network will be wirelessly connected to the network via satellite. In this research the researcher has coined a concept of "Hospital 
Virtualization" that means a hospital running on a virtual network and it is possible via ESXi server. After when the research is built and ready to run an organization so it is necessary to share the results in screenshots for the evaluation with that whole network configuration is also shared on the basis of which actual network is built. The crux of this research is visible as stated in the "Hospital Virtualization Model" with that further are some of the screenshots of the visible results of this research that are conducted and achieved from the simulation within a single host hypervisor without any hurdles that can cause network failure.

The researcher of this research has given a concept; in future it is possible that a network of a big organization might just be in a small pocket size device that might be called a "Pocket Network." This research has further implications in developing actual network on virtualization. So, for future if researchers find themselves inclined towards this study, they can further investigate how actual virtual networks can work in the system of different hospitals et cetera. Virtual networks have the ability to ensure system of cost effective and simple work where it becomes easy for a network engineer to trouble shoot the problems very easily and keep the process running smoothly to avoid any inconvenience.

\section{Health Care Hospital; Network Management System Topology}

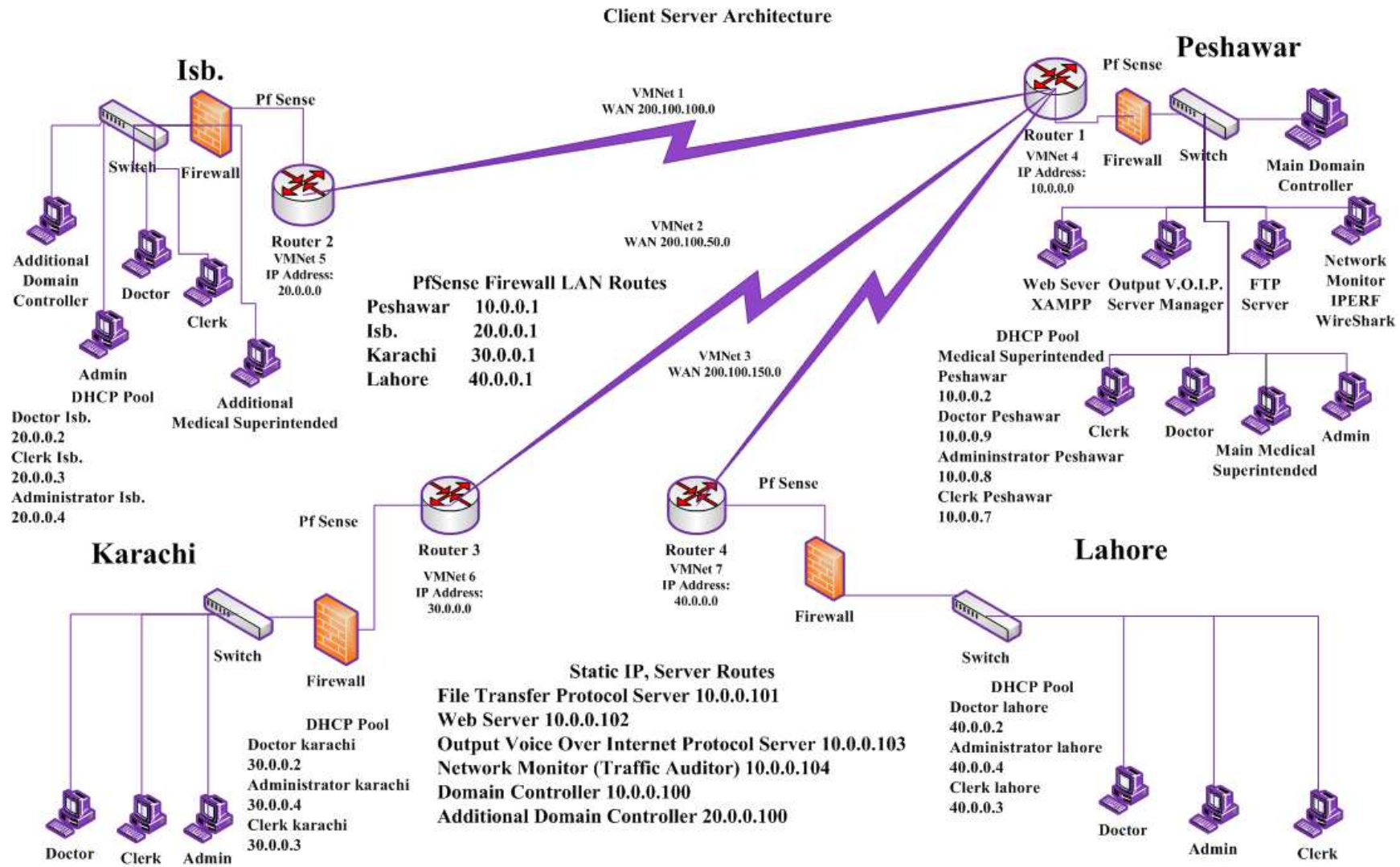

Figure 7. Network Configurations with Network Management System Topology.

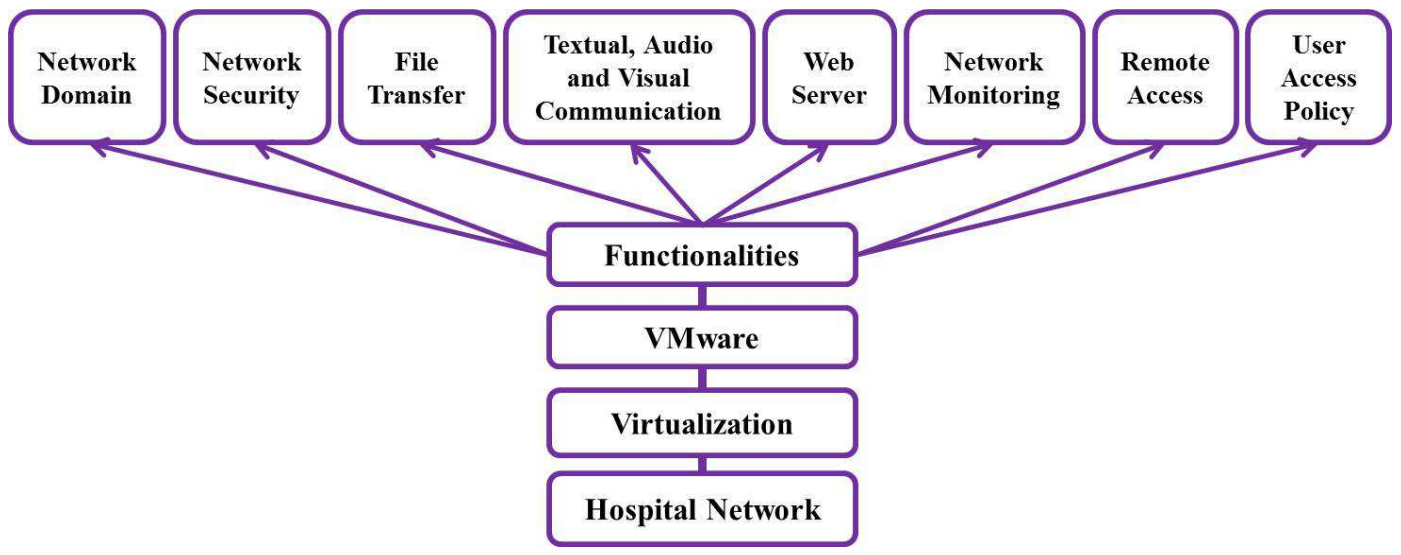

Figure 8. Hospital Virtualization Model. 


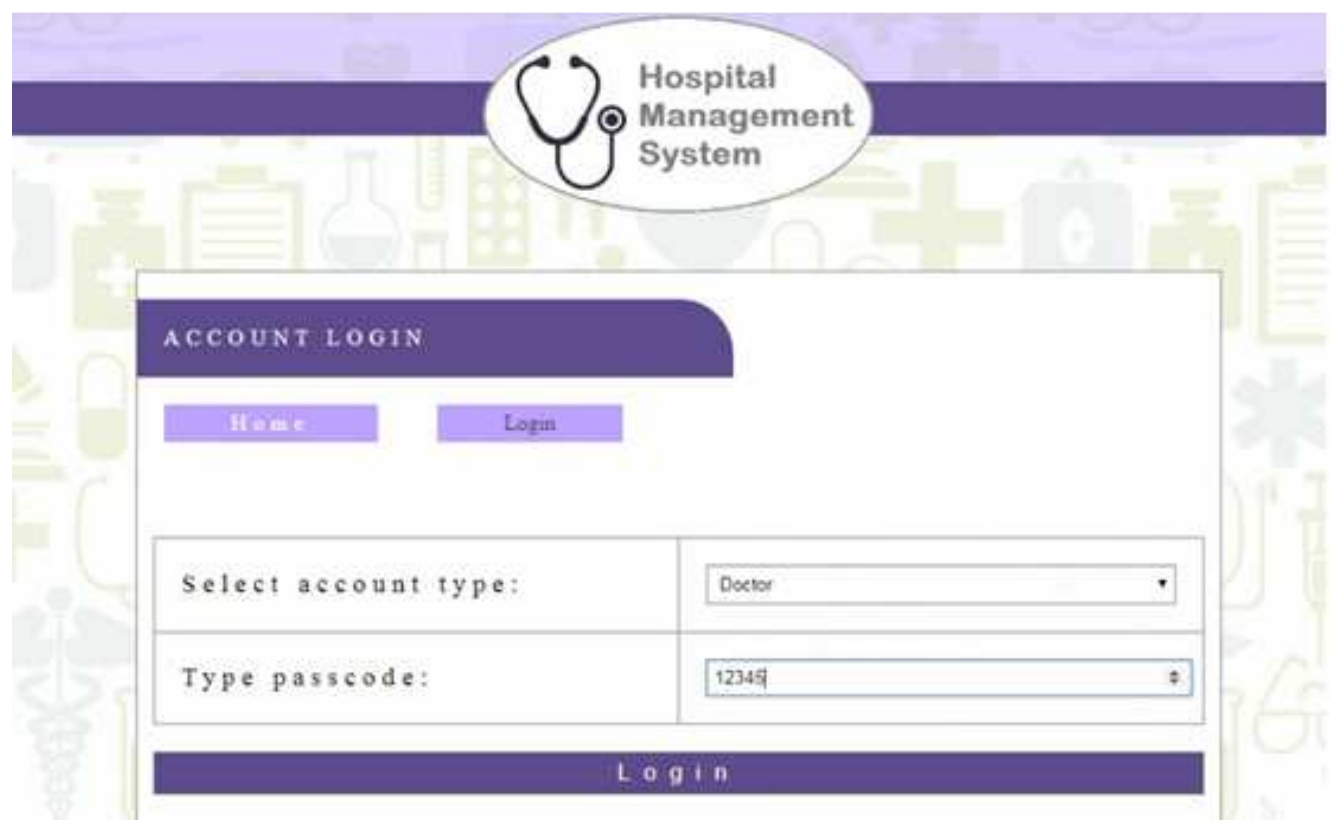

Figure 9. Login Page; Web server Health Care Hospital.

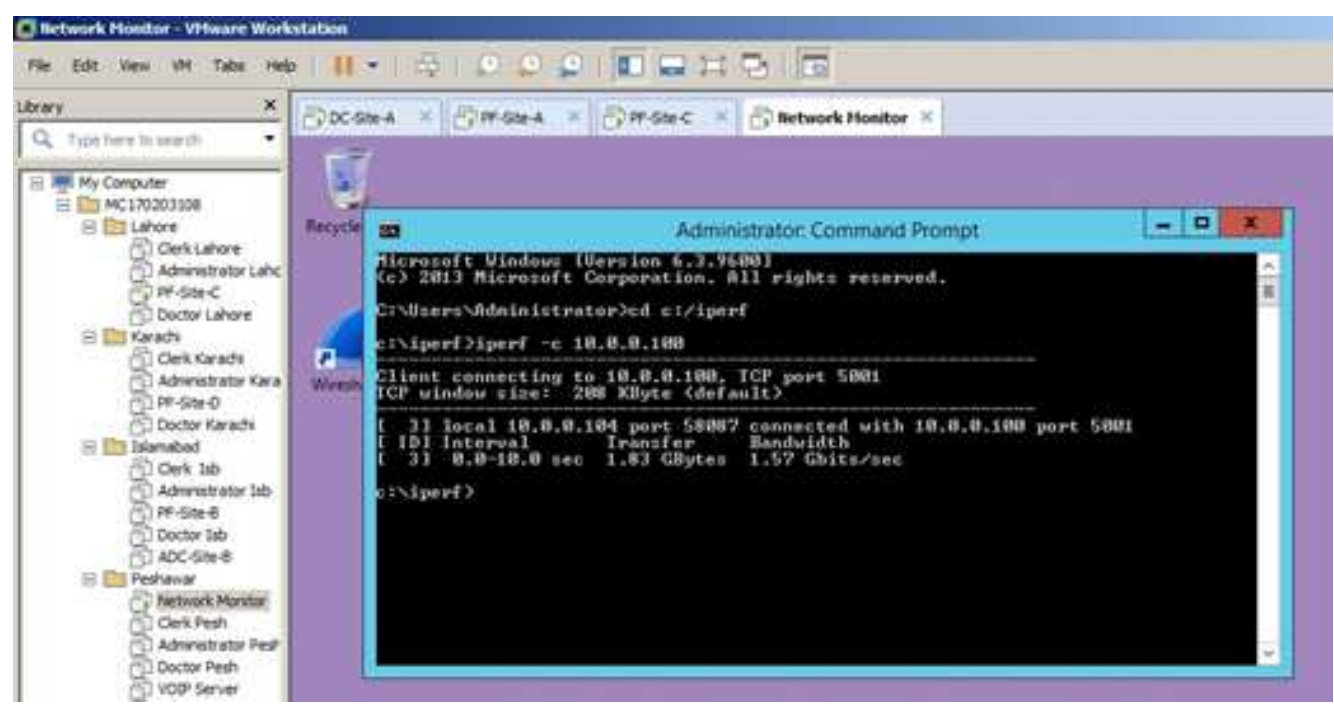

Figure 10. IPERF Tool Screen Network Monitor for Traffic Viewing.

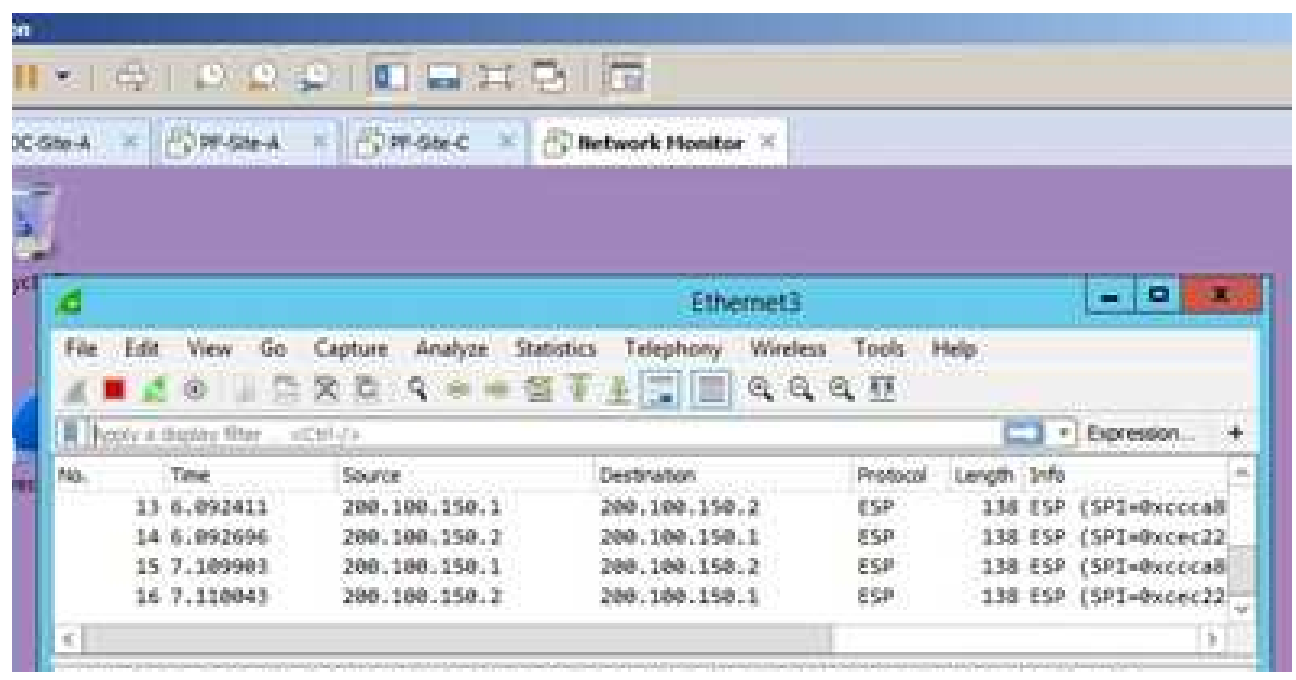

Figure 11. WireShark in Network Monitor for Firewall Encryption View. 
Umair Shahzad et al:: Design, Structure and Significance of Virtual Machines in Network Management System: An Emulation Analysis of VMware Based Hospital System in Pakistan

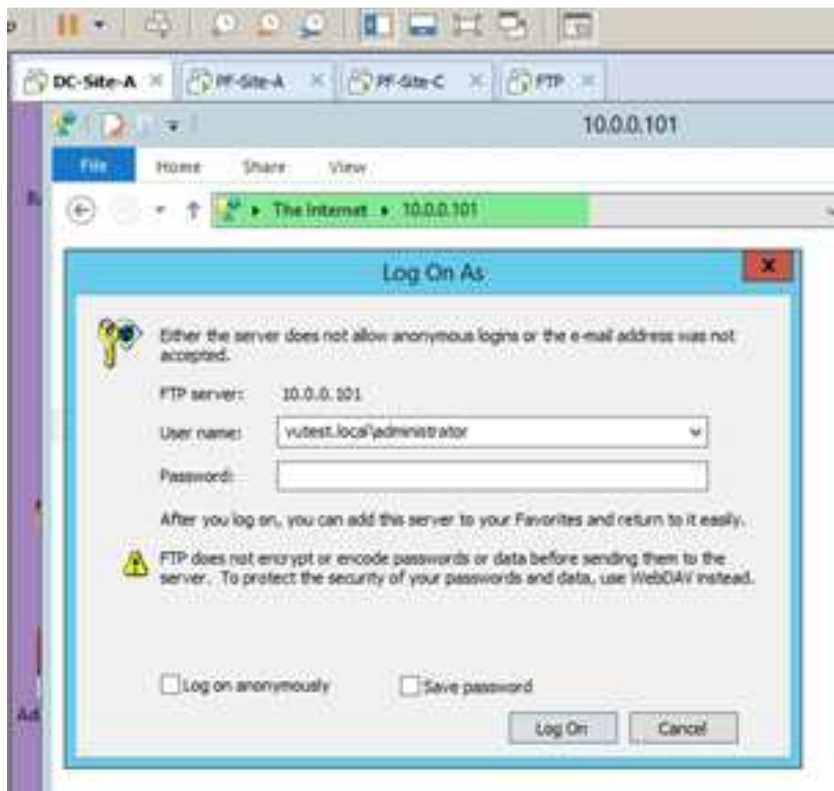

Figure 12. File Transfer Protocol Login View in Domain Controller.

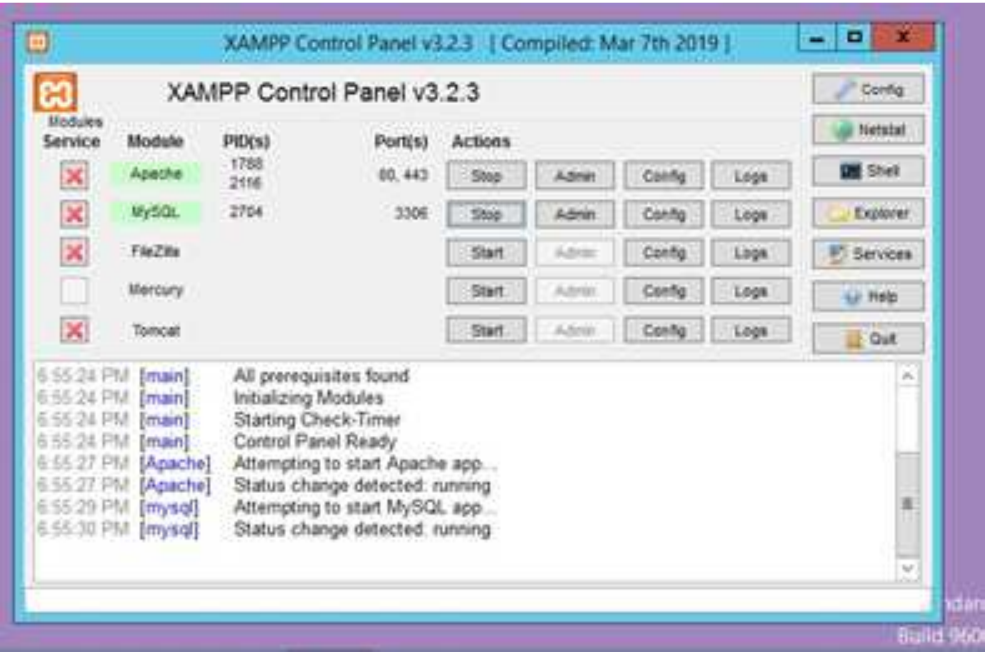

Figure 13. Web Sever XAMPP View.

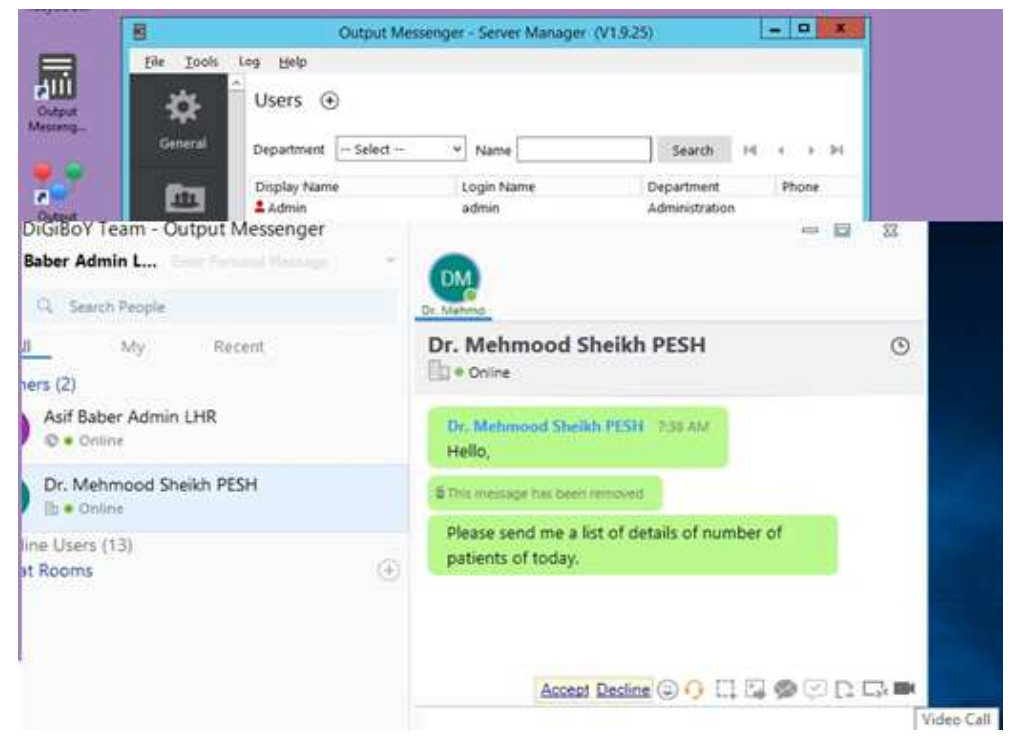

Figure 14. Output Voice Over Internet Protocol Server GUI View. 


\section{Acknowledgements}

This research article is dedicated to most of those who are fighting against the global pandemic; COVID-19 and a peaceful country Pakistan. Research is a path to enlightenment. A small bulb of publication can vitally assist in reducing darkness of unawareness within a country. May peace prevail in Jammu and Kashmir! Indian extremist forces must stop brutality in the area of Jammu and Kashmir! This research article is generated with the satisfactory, innocent, formal and legal efforts of the students Umair Shahzad and Rao Muhammad Adnan, the supervisor Muhammad Luqman and those who were involved in this research including external evaluator et cetera. This simulation-based research is written for academic cause and it is for sharing information among researchers that most of Pakistani researchers are dedicated, hardworking and they have the ability to generate milestones in the area of research if authentic opportunities are provided. "We are one Nation; Pakistan, Jammu \& Kashmir, and Bangladesh.” Quaid-e-Azam-Muhammad Ali Jinnah's Vision.

\section{References}

[1] What is a Network Management System (NMS)?-Definition from Techopedia. (n.d.). Retrieved March 30, 2019, from https://www.techopedia.com/definition/11988/network-manage ment-system-nms

[2] Watt, A. (2014, August 15). 9 scope planning project management. Retrieved April 1, 2019, from https://opentextbc.ca/projectmanagement/chapter/chapter-9-s cope-planning-project-management

[3] A Guide to Networking and Project Management. (2018, July 24). Retrieved from https://www.clarizen.com/a-guide-to-networking-and-projectmanagement/

[4] What is Computer Support?-Definition from Techopedia. (n.d.). Retrieved from https://www.techopedia.com/definition/2645/computer-support

[5] Forthcoming Events. (n.d.). Retrieved from http://www.cs.ucl.ac.uk/index.php?id=4060

[6] VMware Horizon Virtualization Pack for Skype for Business. (2019, April 14). Retrieved from https://www.vmware.com/products/horizon/skype-for-business. html

[7] Introduction to Software Engineering/Process/Methodology. (n.d.). Retrieved from https://en.wikibooks.org/wiki/Introduction_to_Software_Engin eering/Process/Methodology
[8] GNS3. (n.d.). Adding VMware VMs to GNS3 Topologies. Retrieved October 30, 2019, from https://docs.gns3.com/1u_D9XSSA5PVFrOrTWSw1Vn8Utvim d6ksv76F7731N84/index.html

[9] Cassongo, A. B. (2016). The comparison of network simulators for SDN. Новітні інформачійні системи та технологї-Modern information system and technologies, 5 .

[10] Chou, T. S., Baker, S., \& Vega-Herrera, M. (2016, June). A comparison of network simulation and emulation virtualization tools. In Proc. ASEE Annu. Conf. Expo. (pp. 1-9).

[11] Netgate. (2019). The pfSense Book. Retrieved from https://docs.netgate.com/manuals/pfsense/en/latest/the-pfsense -book.pdf

[12] Li, H., \& Chen, G. (2004, May). Wireless LAN network management system. In 2004 IEEE International Symposium on Industrial Electronics (Vol. 1, pp. 615-620). IEEE.

[13] VMware. (2009). Workstation User's Manual [VMware Workstation 7.0]. Retrieved from https://www.vmware.com/pdf/ws7_manual.pdf

[14] Output Messenger. (n.d.). Retrieved May 2, 2019, from https://www.g2.com/products/output-messenger/reviews.

[15] Techopedia. (n.d.). What is a Computer Simulation?-Definition from Techopedia. Retrieved June 2, 2019, from https://www.techopedia.com/definition/17060/computer-simul ation.

[16] Findwords. (n.d.). What does xampp mean. Retrieved July 2, 2019, from https://findwords.info/term/xampp

\section{Biography}

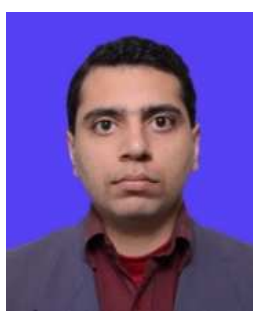

Umair Shahzad He did his Master of Computer Science from Virtual University of Pakistan. He has many distance learning computer science related certifications from Foreign Institutions. He did his M. Phil. Mass Communication Management from The Superior College Lahore. He did his graduation in B. A. (Hons.) major in Mass Communication from Forman Christian College (A Chartered University) Lahore, Punjab, Pakistan. His major research affiliations are mass communication, education, computer science, health, STEM, universe, peace, human health, academics, space and life science. The researcher works for peace in Pakistan and wants to stop brutality in Jammu and Kashmir by India. 